\title{
REMOTE SENSING OF HIGH TEMPERATURE EVENTS BY THE FIREBIRD MISSION
}

\author{
E. Lorenz ${ }^{\mathrm{a}^{*}}$, S. Mitchell ${ }^{\mathrm{a}}$, T. Säuberlich ${ }^{\mathrm{a}}$, C. Paproth ${ }^{\mathrm{a}}$, W. Halle ${ }^{\mathrm{a}}$, O. Frauenberger $^{\mathrm{b}}$
}

German Aerospace Center

a DLR Institute of Optical Sensor Systems, D-12489 Berlin, Rutherfordstraße 2

(eckehard.lorenz, simon.mitchell, saeuberl, carsten.paproth, winfried.halle)@dlr.de

b DLR Institute of Communications and Navigation, D-17235 Neustrelitz, Kalkhorstweg 53 (olaf.frauenberger@dlr.de)

Eckehard.Lorenz@dlr.de

KEY WORDS: Small Satellite Constellation, Infrared Instruments, High Temperature Events, impact on the climatic processes, BiSpectral Method, Advanced On Board Processing

\begin{abstract}
:
More than 10 years after the launch of DLR's first small satellite BIRD, a follow on project called FireBird was started. Based on the success of the BIRD mission, the main scientific goal- the investigation of high temperature events and their impact on the climatic processes- will be continued but in consideration to the advantages given by the operation of a constellation of two small satellites. The first of these satellites- TET-1- was launched on June 22nd 2012. The launch of the second satellite- BIROS- is scheduled for spring 2016.

Both satellites are mainly dedicated to the observation and analysis of high temperature events such as wildfires and volcanoes. The outstanding feature of the FireBird Infrared Instruments is their higher ground sample resolution and dynamic range compared to systems such as MODIS. This enables the detection of smaller fire events and improves the quality of the quantitative analysis.

The analysis of the high temperature events is based on the Bi- Spectral Method, which requires also an excellent characterization of the background temperatures. With this the FireBird Infrared Instruments are also suitable to study phenomena with lower temperatures.

Following the experience of BIRD, the design of the camera system in the visible bands was changed and with this altering the characteristics of the Bi- Spectral Method. These changes were validated in several experiments and the results will be discussed in this paper.

To overcome some restrictions of the small satellite technology, advanced on board processing will be implemented on the FireBird satellites. By implementing the Bi- Spectral Method on board, it is possible to reduce the data stream to a dedicated list of detected high temperature events containing the parameter analyzed. This allows more efficient management of the on board memory and of the downlink capabilities considering also the demand to download selected image data.
\end{abstract}

\section{INTRODUCTION}

FireBird is defined as a constellation of two small satellites mainly dedicated to the investigation of high temperature events. The first satellite TET-1 was launched on12. June 2012. The second satellite BIROS was originally planned to launch in autumn 2015. This date was shifted recently by the launch provider to spring 2016.

Both satellites have identical infrared payload specially designed for detection and measurement of high temperature events in sub-pixel resolution. The payload design is depicted in Figure 1 and Figure 2, with the main parameters shown in Table 1.

Compared to TET-1 the BIROS Satellite will be equipped with a propulsion system to ensure an optimal constellation orbit. Furthermore BIROS will have a much more powerful data handling capability allowing the implementation of very flexible processing chains on board the satellite. With this it is possible to serve quite different user scenarios.

Due to the limited resources of small satellites, some inherent restrictions can be partly avoided by flexible operation of the satellite. A well-developed 'Service on Demand', especially in case of BIROS can markedly improve the data throughput of the satellite.
All these aspects of the usage of a small satellite for the investigation of high temperature events will be discussed in the following document.

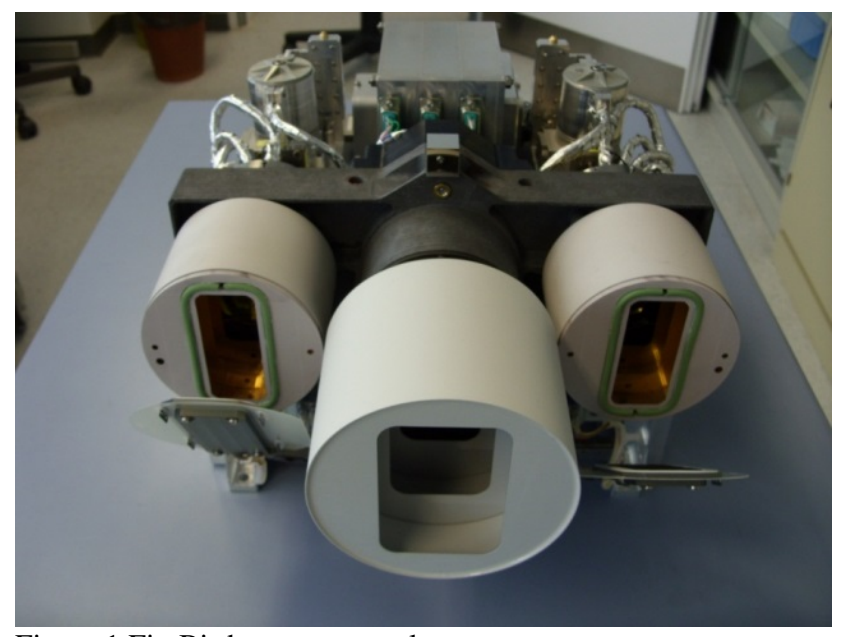

Figure 1 FireBird camera complex 


\begin{tabular}{|c|c|c|}
\hline & $\begin{array}{l}3 \text { line-VIS Camera } \\
\text { ( } 3 \text { line FPA) }\end{array}$ & 2 Infrared- Cameras \\
\hline Wave length & $\begin{array}{l}1460-560 \mathrm{~nm} \\
2565-725 \mathrm{~nm} \\
3790-930 \mathrm{~nm}\end{array}$ & $\begin{array}{l}\text { MWIR: 3,4 - 4,2 } \mu \mathrm{m} \text {; } \\
\text { LWIR: 8,5-9,3 } \mu \mathrm{m}\end{array}$ \\
\hline Focal length & $90,9 \mathrm{~mm}$ & $46,39 \mathrm{~mm}$ \\
\hline FOV & $19,6^{\circ}$ & $19^{\circ}$ \\
\hline F-Number & 3,8 & 2,0 \\
\hline Detector & CCD- Line & CdHgTe Arrays \\
\hline Detector cooling & Passive, $20^{\circ} \mathrm{C}$ & Stirling, 80 - $100 \mathrm{~K}$ \\
\hline Pixel size & $7 \mu \mathrm{m} \times 7 \mu \mathrm{m}$ & $30 \mu \mathrm{m} \times 30 \mu \mathrm{m}$ \\
\hline Number of Pixel & $3 \times 5164$ & $2 \times 512$ staggered \\
\hline Quantization & 14 bit & 14 bit \\
\hline Ground resolution & $42,4 \mathrm{~m}^{2)}$ & $356 \mathrm{~m}^{2)}$ \\
\hline GSD & $42,4 \mathrm{~m}^{2)}$ & $178 \mathrm{~m}^{2)}$ \\
\hline Swath width & $\begin{array}{l}211 \mathrm{~km}^{2)} \\
\mathrm{km}\end{array}$ & $178 \mathrm{~km}^{2)}$ \\
\hline Data rate & $\max 44 \mathrm{MBit} / \mathrm{s}$ nom 11,2 & 0,35 MBit/s \\
\hline Accuracy & $100 \mathrm{~m}$ on ground & $100 \mathrm{~m}$ on ground \\
\hline
\end{tabular}

Table 1. Main FireBird camera parameters

\section{REMOTE SENSING OF HIGH TEMPERATURE EVENTS WITH SMALL SATELLITES}

Remote Sensing to the detection of High Temperature Events (HTE), different aspects have to be considered (Lorenz, 2013).

The temporal and spatial development of a high temperature event may be quite different, but also the intensity of the events, including their background, which has to be considered for the analysis. In (Oertel, 2005) different scenarios were examined with respect to satellite instruments existing at the time. Different observation scenarios will not only be raised by the different nature of the HTEs, but also by the different types of end-user groups as described in (Oertel, 2005). In this context, it is worth continuing the discussion of the advantages and disadvantages of small satellites as started in (Lorenz, 2013).

For the investigation of such high dynamic events like bush fires, the revisit time and the over flight time of the satellite is an important assessment criterion. Due to the piggy back launch of small satellites, the possibility choosing the over flight time is minimal. The probability to get an ascending node around 12:00 is very low. TET and BIROS have a crossing time around 9:30.

With regards to the revisit time, the variability is higher. In a standard flying mode the revisit time of TET- 1 is approximately 3 or 4 days. Assuming a second satellite like BIROS this could be reduced, depending on the concrete orbit parameter of BIROS, by up to 1 day.

Additionally, the high agility of the satellites has to be considered, with tilting $+/-30^{\circ}$ across the track available. Considering that the nadir position of line of sight differs from day to day around $600 \mathrm{~km}$, it is possible to arrange by tilting the satellite an overlap of the swath of two subsequence days in the order of $100 \mathrm{~km}$. With this it is possible to observe with one satellite a HTE in two subsequent days and skip the third day. With a second satellite it would be possible to guarantee more than a daily coverage for a certain target.

The previous paragraph considered only a day time or a night time passage. When combing both (see Figure 3) it is possible, depending on the concrete orbit constellation, to get up to two images of the same target per day. This could be demonstrated in different cases.

A very important aspect in context with the small satellites is the data volume to be stored on board and down linked to Earth. Due to the limited resources, small satellites - so also TET and BIROS- are equipped mostly with an S- Band transmitter. This allows transmitting approximately $100 \mathrm{MB}$ per contact. The 5 spectral bands of FireBird generate more than $100 \mathrm{MB}$ and so more than one contact are necessary to transfer the data completely.

In regards to the observation of HTEs, a solution to the problem could be achieved in the thematic data volume reduction. As demonstrated with BIRD (Briess, 2003), it is possible to reduce the information regarding a fire event to a minimum of the main parameters as shown in Table 3. This allows for a reduction of the data volume to be stored and down linked later to a minimum. Of course it is necessary to agree with the community which data sets can be reduced and which has to be preserved unchanged for further investigations.

An alternative or a supplement is of course the integration of additional ground stations preferably in the Polar Regions. Currently, different opportunities are in discussion, but as yet there are no firm plans for implementation.

\section{BIROS}

The BIROS Satellite (Bi-spectral Infrared Optical System) is based on DLR proven approach developed for the BIRD (Bispectral Infrared optical System) mission, which was launched in 2001 (Briess, 2003). The BIROS Satellite Bus segment has the same technology as the TET-1 satellite, which was launched successfully in July 2012 as the first German "Technologie Erprobungs Träger“ (TET) satellite. TET-1 was initiated and funded by the DLR Space Agency as one element of the German On-Orbit Verification (OOV) Program. In the end of 2013 TET-1 was handed over to the FireBird mission.

BIROS' primary payload is the same multispectral camera system as on the TET-1 satellite (see Figure 2). Also on board of BIROS other new technological experiments related to scientific and technological challenges of next generation remote sensing satellites will be installed. Especially in the field of space born disaster warning systems, micro satellite systems are becoming more flexible, for example highly agile and fine pointing platforms suitable for swath width extension or in track stereo, fast target pointing, high commanding flexibility of the sensor systems in order to have different data take scenarios and finally a fast and flexible information distribution to the end user on ground.

Additionally, BIROS will contain a technical experiment using a hardware VHF modem, whereby communicating with an ORBCOMM satellite (altitude $800 \mathrm{~km}$ ), it will be possible to inform directly the ground users via E-Mail and SMS about an on-board detected hot-spot with the concerning geo-location (Table 3). This also opens the way for a flexible on-board planning (software experiment). In case of a successful 
assessment of this experiment it will be established as a standard procedure improving with this the flexibility of data acquisition (Halle, 2014).

\section{OPERATION AND DATA PRODUCTS}

The operation of small satellites is different from larger satellites for reasons discussed in section 2. For the users this generates the advantage of a 'service on demand', but because of limited resources, this requires a very dedicated selection of the data take targets. In this context, an individual ordering of the data takes by a restricted group of people is necessary. The users can delegate their requests to the ordering group and in case of conflicts will be decided about the priority.

Besides the urgency to gather information about a disaster situation, the terms conflict and priority are dependent mostly on technical parameters like data volume to be stored and down linked and the number of available ground stations.

The planning of the data takes will be supported by an efficient ordering tool called SPOT which was developed by DLR GSOC. The GUI of SPOT is shown in Figure 3.

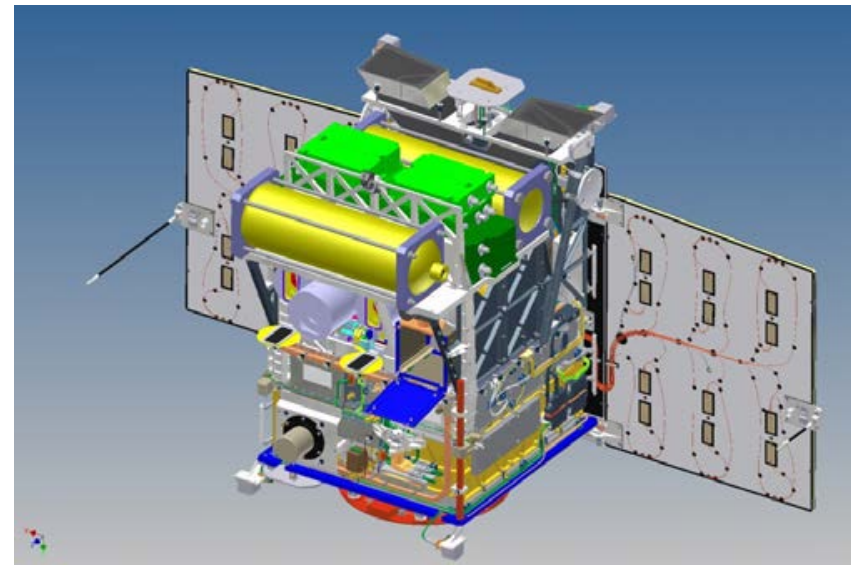

Figure 2 BIROS Satellite with the Payload Segment

With SPOT there is also the possibility of predicting future data takes and with this a dedicated planning of burning experiments and other projectable events will be well supported. In August 2014 TET-1 supported an international experiment with prescribed burning in the Krueger National Park in South Africa (Govender, 2014; Figure 11).

This was also a good opportunity to validate as well the existing TET-1 procedures.

The user can choose from four standard instrument configurations which were mainly establish to save on board memory capacity (Table 2). During the night vision measurements (Fire Night) the visual bands can be switched off. At day the GSD of the visual bands can be switched between $40 \mathrm{~m}$ and $160 \mathrm{~m}$ and VIS1 can select which of the visual bands should be transmitted to Earth. In the consequence this is a decision between the GSD and the area to be monitored.

\begin{tabular}{|c|c|c|c|c|c|c|c|c|c|}
\hline Mode & MWIR & MWIR-CAL & IWIR & IWIR-CAL & VISN & VISR & VISG & AOCS & Remark \\
\hline Fire $4 \times 4$ & $x$ & $x$ & $x$ & $x$ & $x$ & $x$ & $x$ & $x$ & VIS GR $-160 \mathrm{~m}$ \\
\hline FireNight & $x$ & $x$ & $x$ & $x$ & & & & $x$ & \\
\hline VIS1_backward & $\mathrm{x}$ & $x$ & $x$ & $x$ & $x$ & & & $\mathrm{x}$ & VIS GR \\
\hline VIS1_forward & $\mathrm{x}$ & $x$ & $\mathrm{x}$ & $x$ & & & $x$ & $x$ & VIS GR $=40 \mathrm{~m}$ \\
\hline VIS1_nadir & $x$ & $x$ & $\mathrm{x}$ & $x$ & & $x$ & & $x$ & VIS $G R^{-4}-40 \mathrm{~m}$ \\
\hline VIS3 & $x$ & $x$ & $x$ & $x$ & $x$ & $x$ & $x$ & $x$ & VIS $G R^{-40 m}$ \\
\hline Systemorder & $x$ & $x$ & $x$ & $x$ & $x$ & $x$ & $x$ & $x$ & VIS $G R^{-}=40 \mathrm{~m}$ \\
\hline
\end{tabular}

Table 2. TET-1 Standard Mode Configuration
It has to be considered that the setting of an order is no guarantee for an execution because of possible on board memory conflicts with previous data takes. All handling of FireBird data is described in the Data Policy Document.

Receiving the raw data, an operational processing unit generates a standard format for the raw data. This level 0 data set includes -depending on the operation mode- up to five measurement files, two calibration files for the infrared cameras and an attitude file. The last file types are of less interest for the users.

Based on these raw data files the L1b standard products will be generated. L1b products are radiometric calibrated radiances top of atmosphere with geographic annotation and related Meta Data Information. This information can be delivered in an ENVI conform format with additional XML files or in a HDFEOS-5 format.

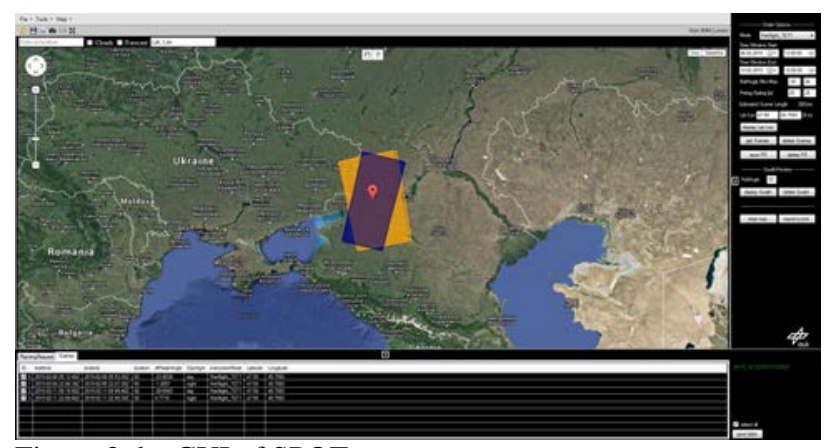

Figure 3 the GUI of SPOT

After the successful execution of an order including processing to $\mathrm{L} 1 \mathrm{~B}$, the products are transferred to an FTP server and the user will be informed that they can download the products per FTP.

Meta information generated during L0 pre-processing and level $1 \mathrm{~b}$ processing as well as level $1 \mathrm{~b}$ quick looks will be added to the level 0 product and archived. Valid products will be registered in the DLR EOWEB Catalogue. Here registered user can search for products of interest and in case of success place orders for level $1 \mathrm{~b}$ products. These products will be reprocessed from the archived L0 products. This ensures that data provided are processed with the most recent version of the data processor.

\section{RADIOMETRIC CALIBRATION AND VALIDATION}

In order to obtain scientifically usable image data, a radiometric calibration of the sensor's visible and infrared channels has to be performed. The application of the corresponding calibration data sets on raw image data is the first step in the image processing pipeline and aims for the conversion of the digital raw image data into units of mean spectral radiances related to each channel's spectral band. For the visible (VIS) / near infrared (NIR) channels calibration datasets have been determined from on-ground flat-field measurements using well characterized reference sources. These datasets will be applied to the incoming VIS/NIR raw data. For the mid wave (MWIR) and long wave infrared (LWIR) channels, in-flight calibration data will be recorded after the data take.

The calibration procedure in case of FireBird is not a classical two point method but is based on a correlation of the detector signals with continuously heating process of the black flap in front of the IR optics (see Figure 4 and Figure 5). 
These efforts focus on the development of algorithms for the reconstruction of scene signal portions with a very high signal dynamic. For example very hot or extended hot events on relative cold backgrounds (Figure 6) can show in the MWIR and LWIR channels non-linear signal responses especially for very low signal levels or even information loss due to distortion. The described calibration procedure can check the linearity of the signal responses and based on the knowledge of the spatial distribution of the incoming radiation information within the images (PSF), lost information can be at least partially estimated or reconstructed respectively. The result is an increase of the effective dynamic range of the sensor channel. Additionally, the signal dynamic range can be increased by operating the system in a special mode with a reduced integration time for very hot scenes.

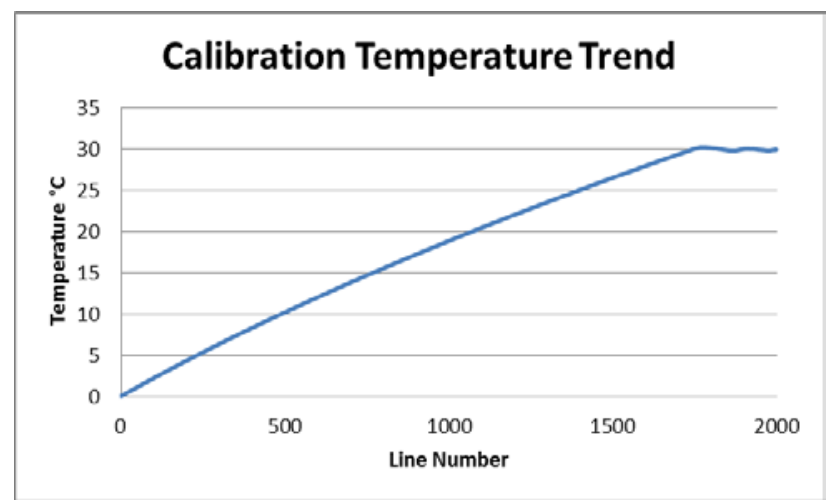

Figure 4. Calibration Flap Temperature trend

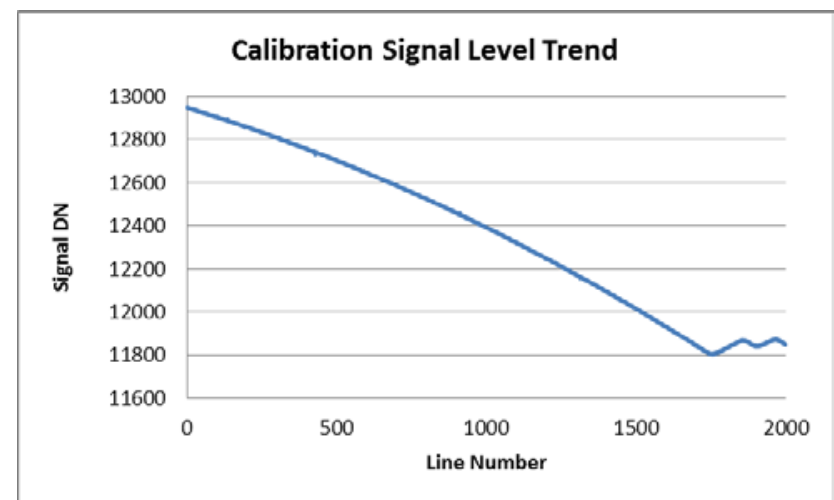

Figure 5 Detector signal correlated to the Flap Temperature

\section{HOT AREA TECHNOLOGY AND THE BI- SPECTRAL ALGORITHM}

The observation of high temperature events and especially of wild fires causes strong requirements to the dynamic range to be overcome by the instrument. An extreme example is the observation of the Bardabunga Volcano in Iceland (Figure 6), especially in the MWIR Band. In this image there is ice and fire side by side and the fire fills a number of detector pixels, which requires a very high saturation temperature. On the other side, the low IR signal of the glacier forces the detector in the nonlinear operating range as described above. Both extremes can be handled with extremely different integrations intervals of the infrared detectors. That is exactly the technology which is implemented in the IR cameras of FireBird. The dwell time for the IR cameras is approximately $20 \mathrm{~ms}$, the integration interval for background temperatures $\sim 20^{\circ} \mathrm{C}$ is set to $4 \mathrm{~ms}$. Controlling during the read out process in real time the signal levels of the first standard exposure, in the case of saturation, it is possible to initiate a second exposure with a much shorter integration interval. This technology allows for the covering of an extremely high dynamic range.

In case of a very small hot spot which covers only a part of an image ground pixel, the detector signal is a mix of the background temperature and the high temperature resulting in a brightness temperature in the order of $40^{\circ} \mathrm{C}$. For calculation of the hot spot temperature from this brightness temperature the Dozier method (Dozier, 1981) will be applied.

In the single pixel case, the effective fire temperature $T_{F}$ and the fire proportion in the pixel $\mathrm{q}_{\mathrm{F}}$, which is related to fire area $\mathrm{A}_{\mathrm{F}}$, are found by solving the mixing equations for the pixelaveraged radiance in two channels:

$$
I_{j}=q_{F} B_{j}\left(T_{F}\right)+\left(1-q_{F}\right) I_{j, b g}^{k}
$$

where $\mathrm{I} j$ is the atmospherically corrected radiance of a hot pixel in channel $j$ ( $j=$ MIR and TIR), $B_{j}\left(T_{F}\right)$ is the black-body radiance in channel $j$ as a function of temperature $T, I_{j, b g}$, is the radiance of the non-fire portion of the hot pixel that has to be estimated from the neighboring background pixels.

Since the MIR radiance emitted by a fire is so intense, even smaller sub-pixel fires are likely to significantly affect the radiance signal of not only the pixel within which the fire is actually located, but also the signal of neighboring pixels. This effect is especially pronounced in TET-1 images due to the double sampling causing pixels to overlap by $50 \%$. For this reason, active fires are usually recognized as clusters of 'hot' pixels in the MIR imagery, here termed 'hot clusters'.

The area of any hot cluster in an image should not be confused with the area of the causal fire. The column 'size' in Table 3 indicates how many pixels are included in the cluster described by a row of this table.

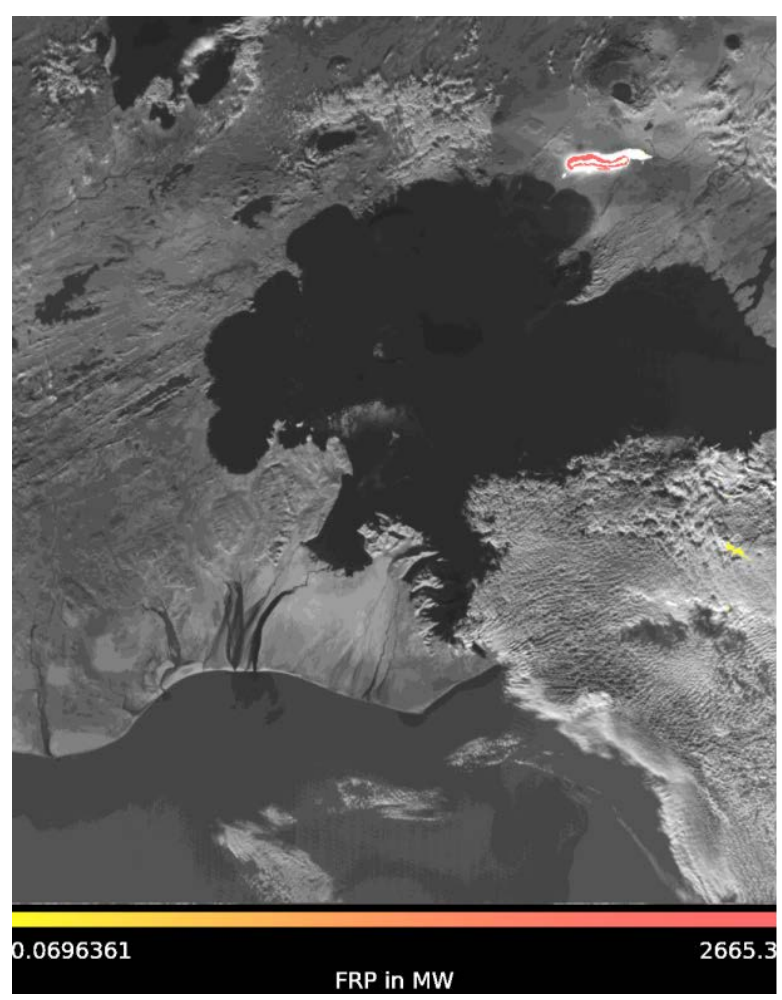

Figure 6. Fire and Ice- The Bardabunga Volcano in Iceland 


\section{USAGE OF THE SATELLITE ATTITUDE DATA}

The usage of the Satellite Attitude Data has quite different aspects.

Firstly it is necessary to locate the detected fires exactly, especially in case that the extended on board processing will be used as described in section 2. This will be provided by calculating the coordinates directly using the Satellite Attitude Data. This is a demanding task. An uncertainty of $1 \mathrm{arcmin}$ in the overall pointing resulting from the camera pointing in the satellite and the satellite itself leads to a localisation error of $145 \mathrm{~m}$ on ground. This is in the order of one IR ground pixel.

But also an interactive ground processing by an indirect geo referencing using reference control points is not always possible, as shown in Figure 7. When not having the direct geo referencing, the long term investigation of events such as the peat fires in cloudy region would be difficult. On the other hand Figure 7 shows also, that cloudiness is not automatically a criterion to classify an image as trashy.

Last but not least, quite tricky manoeuvres are possible with a small satellite using the close relation between the camera data and the attitude data. The FireBird satellites are equipped with a high agility thanks to powerful reaction wheels. So it is possible to turn the satellite in a forward looking observation mode to detect targets of interest. In case of the detection of hot spots the satellite can turn quickly in the nadir looking modes and measure this feature in detail. This is also an example of possible data volume management, in taking only the real areas of interest.

\section{TET-1 APPLICATION FOR WILD FIRE MONITORING}

Since beginning of 2014, TET-1 acquired approximately 1200 data takes, many of them over regions with wild fires. In this section, some examples of data images acquired with TET-1 will be shown.

Figure 8 shows the resultant fire products overlaid onto the TET-1 MWIR band for an image taken during the Australian 2014 fire season, which shows a typical example of a large wildfire occurrence. This image was of the Blue Mountain area west of Sydney. Other examples of fire scenarios from Australia are illustrated in Figure 9 and Figure 10. These images detail a fire complex in Southwest Western Australia in February 2015. Comparing Figure 9 and Figure 10 with Figure 8 and with the output from BIRD (Zhukov, 2005), it can be seen that the fire fronts detected in both cases are quite different due to the different fuel material burning.

These images highlight the ability of TET- 1 to detect fires over a large range of temperatures and areas, in both day and night time conditions. Furthermore, Figure 9 and Figure 10 demonstrate the capability to observe short term events with one satellite if the constellation orbit allows. Here was realised a revisit time of 36h, although shorter timespans are possible.

Table 3 shows the FRP Table related to Figure 10. This is an example of the information available for the reduced form which could be sent via SMS by BIROS. Further suggestions for content are welcome.

In October 2014 in Kalimantan, Indonesia large peat fires raged, as documented in Figure 7. This figure shows that the fires can be seen through the hazy fog. This leads of course to an underestimation of the FRP and atmospheric correction would be necessary.

In case of the Kalimantan fires, TET-1 could demonstrate often a revisit time in order of $12 \mathrm{~h}$ using night and day. Within three weeks, many images were taken of the area, which allowed for much data about the progression processes was collected.

The South Africa Experiment in 2014 was mentioned in section 4. This was an excellent opportunity to validate the TET-1 fire products. Besides the ground validation measurement a direct comparison with an alternative FRP model (Wooster, 2003) was possible. It found a good agreement between the TET-1 data and related helicopter measurements

\section{CONCLUSION}

In this paper were demonstrated the capabilities of small satellites to detect and monitor high temperature events. Small satellites can be an alternative to the established, bigger satellites if they will be operated in an adapted mode. Nevertheless the methods implemented to the FireBird instruments can be assigned to any satellite classes.

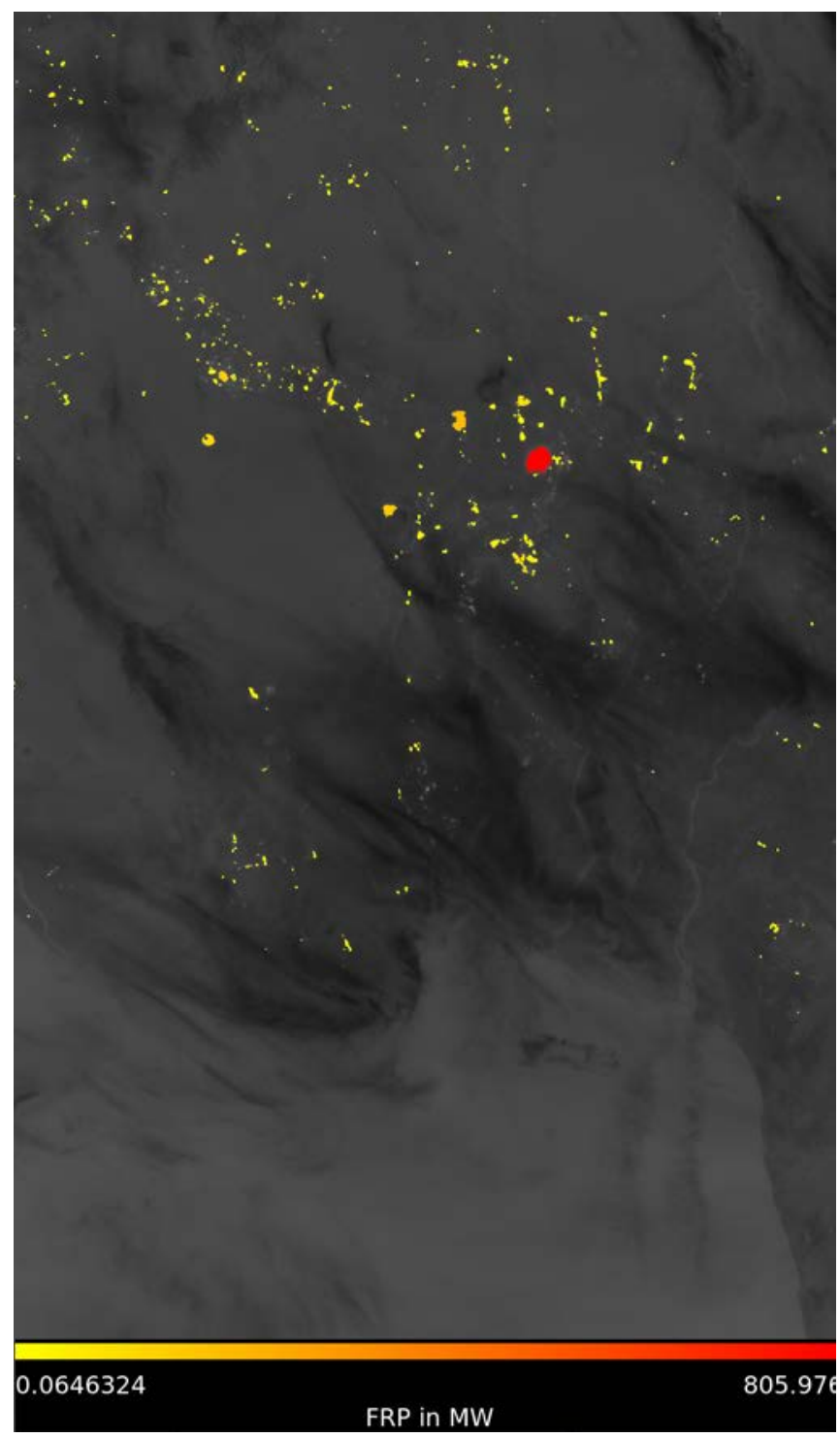

Figure 7. Kalimantan (Indonesia) 09. 10.2014 16:33 UTC 


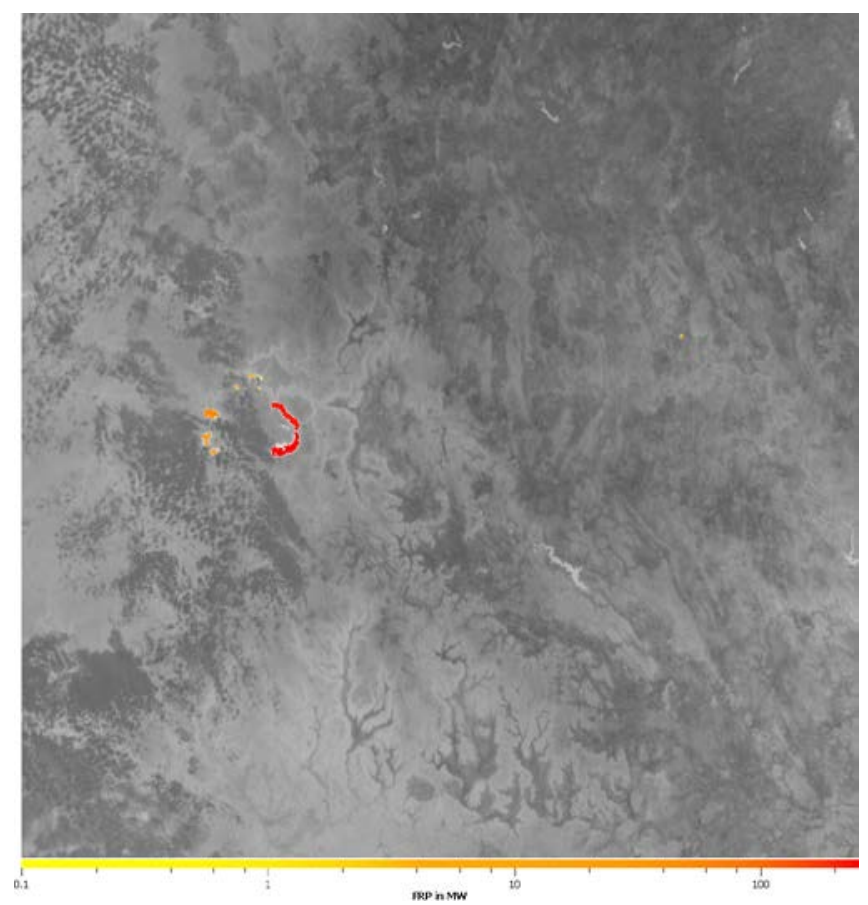

Figure 8. Blue Mountains (Sydney) Region 04.01.2014 13:33 UTC

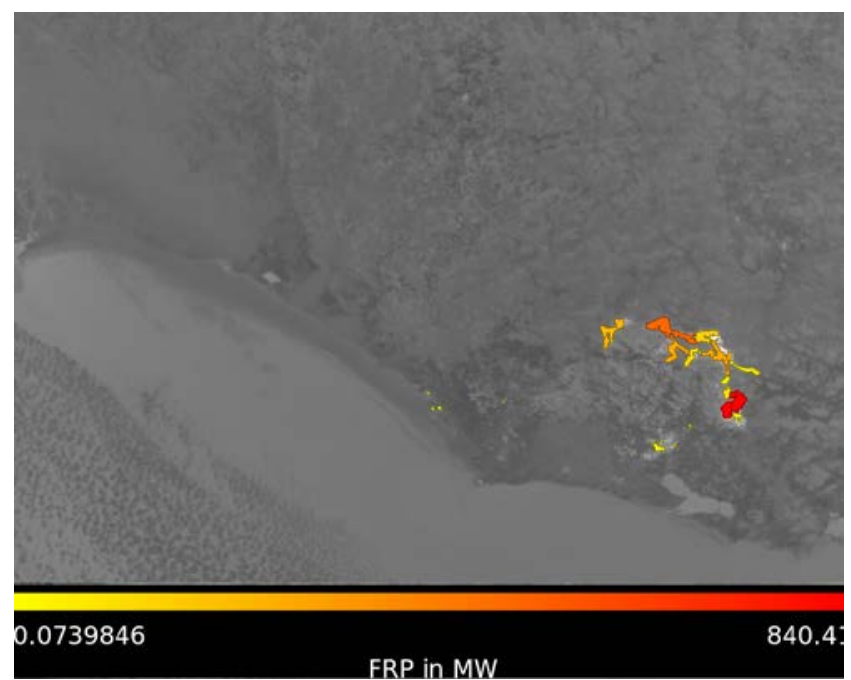

Figure 9 Northcliffe (West Australia) 8.2.2015 16:11UTC

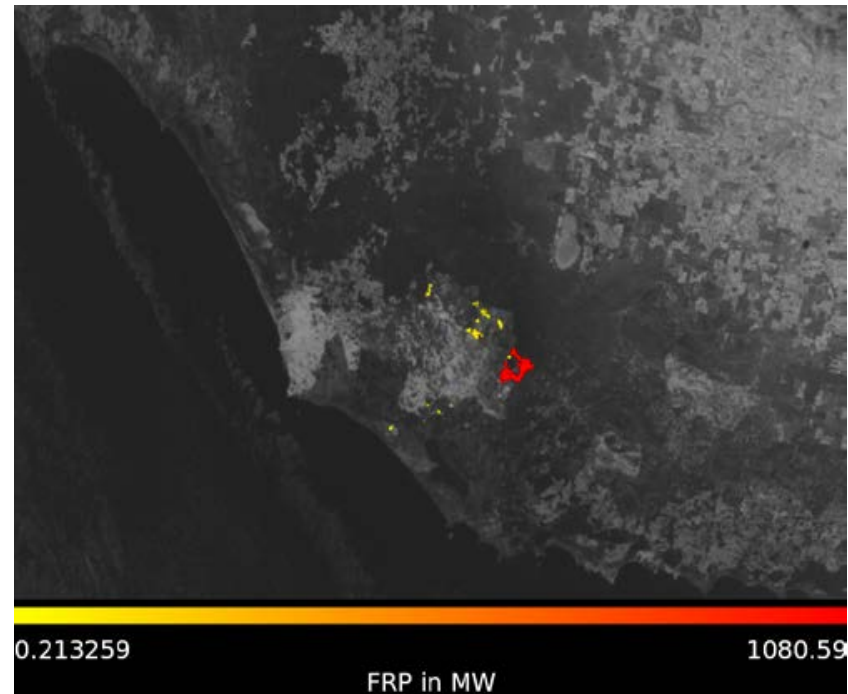

Figure 10 Northcliffe (West Australia) 10.2.2015 04:42 UTC

\begin{tabular}{|c|c|c|c|c|c|c|c|}
\hline size & $\mathrm{x}$ & $\mathrm{y}$ & lat & lon & frp & $\mathrm{T}$ & $\mathrm{A}$ \\
\hline 10,00 & 461,66 & 209,52 & $-34,88$ & 116,29 & 16,96 & 564,25 & 2951,52 \\
\hline 5,00 & 521,62 & 229,18 & $-34,84$ & 116,41 & 8,52 & 637,44 & 909,84 \\
\hline 4,00 & 536,56 & 236,50 & $-34,83$ & 116,44 & 7,08 & 668,50 & 625,38 \\
\hline 5,00 & 507,39 & 237,40 & $-34,83$ & 116,38 & 6,03 & 1500,00 & 21,01 \\
\hline 512,00 & 616,40 & 280,39 & $-34,74$ & 116,59 & 1080,59 & 578,10 & 170626,28 \\
\hline 6,00 & 608,05 & 296,49 & $-34,72$ & 116,57 & 3,37 & 908,39 & 87,31 \\
\hline 64,00 & 566,59 & 324,59 & $-34,69$ & 116,48 & 87,23 & 1500,00 & 303,86 \\
\hline 3,00 & 574,21 & 322,37 & $-34,69$ & 116,49 & 2,60 & 909,41 & 67,06 \\
\hline 13,00 & 558,57 & 329,66 & $-34,69$ & 116,46 & 14,23 & 736,34 & 853,69 \\
\hline 37,00 & 597,04 & 336,63 & $-34,67$ & 116,54 & 52,20 & 648,69 & 5199,15 \\
\hline 8,00 & 569,32 & 340,97 & $-34,67$ & 116,48 & 10,38 & 855,79 & 341,32 \\
\hline 12,00 & 581,69 & 346,42 & $-34,66$ & 116,50 & 9,28 & 1500,00 & 32,33 \\
\hline 20,00 & 576,11 & 350,87 & $-34,65$ & 116,49 & 61,25 & 2897,63 & 15,32 \\
\hline 1,00 & 568,00 & 359,00 & $-34,64$ & 116,47 & 0,49 & 1500,00 & 1,72 \\
\hline 3,00 & 564,64 & 361,31 & $-34,64$ & 116,46 & 1,42 & 918,32 & 35,34 \\
\hline 4,00 & 568,44 & 361,93 & $-34,64$ & 116,47 & 1,92 & 1066,62 & 26,20 \\
\hline 33,00 & 508,47 & 376,64 & $-34,63$ & 116,34 & 31,44 & 743,28 & 1816,48 \\
\hline 1,00 & 221,00 & 2265,00 & $-32,06$ & 115,13 & 0,21 & 1500,00 & 0,74 \\
\hline
\end{tabular}

Table 3. FRP Table for the data take 10.2.2015 04:42 UTC 


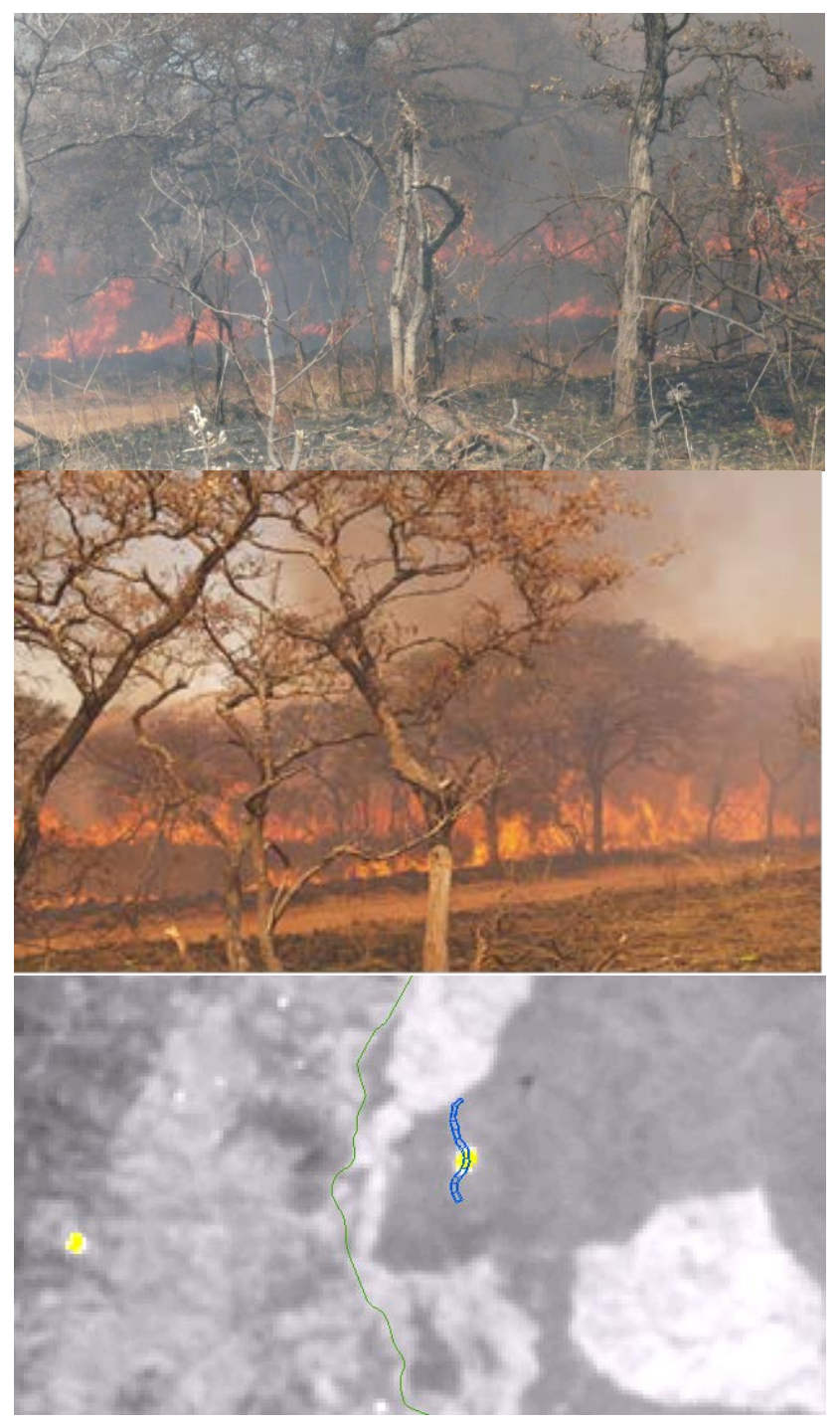

Figure 11 upper: photographs of fire at Shabeni plot No. 3 shortly after TET-1 overpass, Lower: TET-1 satellite image (FRP of fire detections in yellow, background: mid wave -IR channel, National Park boundary in green, plot outlines of Shabeni plot string in blue (origin from Govender, 2014)

\section{REFERENCES}

Briess, K., Jahn, H., Lorenz, E., Oertel, D., Skrbek, W., \& Zhukov, B. (2003). Fire recognition potential of the Bi-spectral InfraRed Detection (BIRD) satellite. Int. J. Remote Sensing, 24, 865-872.

Dozier, J.(1981). A method for satellite identification of surface temperature fields of subpixel resolution. Remote Sens. Environm., 11, 221-229.

Govender, N

KNP Field Validation Campaign

Report - 17th $-31^{\text {st }}$ August 2014

Compiled by Navashni Govender with contributions from Wilfrid Schroeder, Louis Giglio, Bob Kremans, Gernot Ruecker, Olaf Frauenbergen, Martin Wooster, Mark Dejong, Bruce Main, Ronan Paugam, Evan Ellicott and Anja Hoffmann

Halle, W., Bärwald, W., Raschke C., Terzibaschian T.

The DLR -Satellite BIROS in the FireBIRD Mission

4S Symposium 26 May 2014 - Friday, 30 May 2014, Porto Petro, Majorca, Spain

Lorenz, E

Thermal Remote Sensing with Small Satellites: BIRD, TET and Next Generation BIROS

In

Thermal Infrared Remote Sensing

Springer Dordrecht Heidelberg New York London 2013

Editors C. Kuenzer \& St. Dech

Oertel, D

ECOFIRE Study on Scientific Assessment of Space-borne High Temperature Event Observing

Mission Concepts

ESTEC Contract No. 17690/03/NL/FF

Final Report, December 2005

Wooster, M., Zhukov, B., \& Oertel, D. (2003). Fire radiative energy release for quantitative study of biomass burning: derivation from the BIRD experimental satellite and comparison to MODIS fire products. Remote Sens. Environm., 86, 83107.

Zhukov, B., E. Lorenz, D. Oertel, M. Wooster and G. Roberts Experience of detection and quantitative characterization of fires during the experimental small satellite mission BIRD Final Report to the BIRD Mission, DLR 2005 